\title{
Effect of Foliar Application of Seaweed Extract and Zincon Productivity of Dahlia hybrida cv. Albion
}

\author{
Hassan Hadi Alkarawi $^{\text {a }}$ Sabaa Jawad Abd Alkadim ${ }^{\text {b }}$ \\ ${ }^{A, b}$ AlForat Alawsat Technical University, Technical College Musayib \\ hassan2008hadi@googlemail.com_sabajwad78@g.mail.com
}

Submission date:- 3/12/2018 Acceptance date:- 15/1/2019 Publication date:- 7/4/2019

Keywords: Dahlia, Seaweed, zinc sulphate, foliar application, tubers.

\begin{abstract}
An experiment for the agricultural season 2017 was carriedout in pots in the Autumn season to study the effect of application three levels of Sea Top foliar fertilizer $\left(0,50\right.$, and $\left.100 \mathrm{gm} . \mathrm{L}^{-1}\right)$ and Three levels of zinc (zinc sulphate) was $\left(0,30\right.$, and60 mg. $\left.\mathrm{L}^{-1}\right)$ with three times spraying intervals every20 days for some of the growth and yield parameters of the Dahlia cv. Albion. The spray concentration of zinc sulfate at level of $60 \mathrm{mg} / \mathrm{L}^{-1}$ and Seaweed at a level of 100 $\mathrm{mg} / \mathrm{L}^{-1}$ had significant differences in plant height, number of branches ,number of flowers per plant, flower diameter, fresh weight of flower,fresh weight of the tuber, number of tubers per plant, fresh weight of the stalk,and dry weight of the vegetative parts ,compared with the control treatment.The increase was 115.16, 6.33, 12.0, 15.53, 25.66, 371.36, $6.66,46.56$, and 60.16 for the above qualities respectively.

We conclude, that application of Seaweed Extract and Zinc improved the yield contributing factors that resulted in significant increase in Productivity of Dahlia hybridayield.

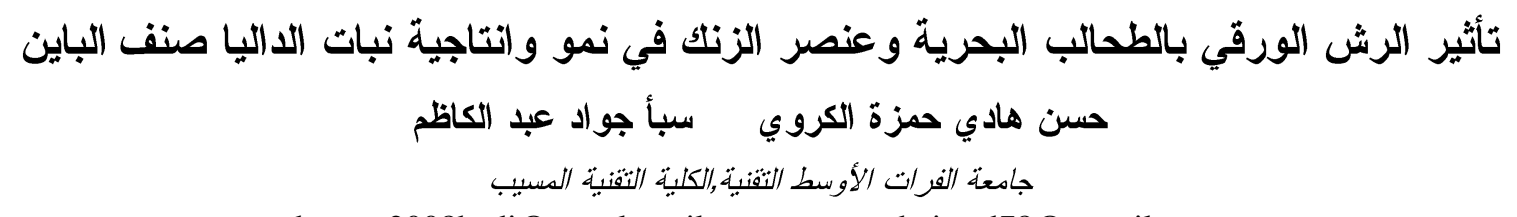
hassan2008hadi@googlemail.com sabajwad78@g.mail.com

\footnotetext{
الخلاصة

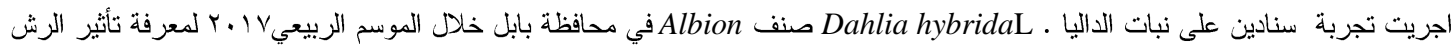

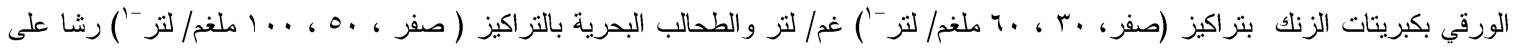

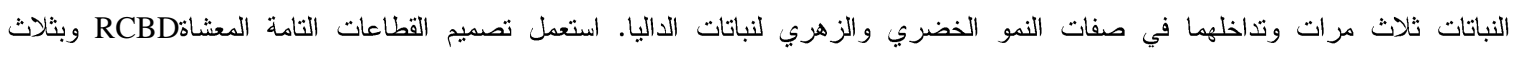

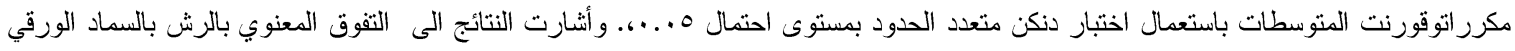

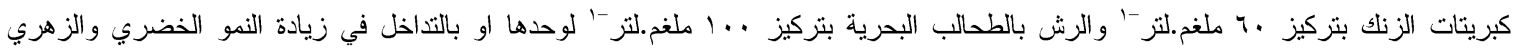

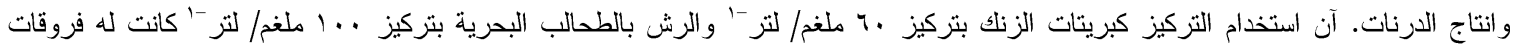

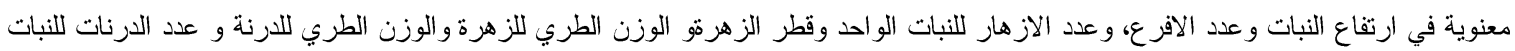

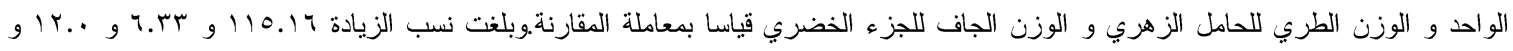

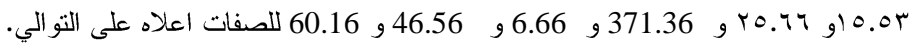
نستتتج ان اضافة الاسمدة الورقية بالرش بعنصر الزنك وان والطحالب البحرية قد اعطى فروقات معنوية في جميع الصفات المدروسة. الكلمات الدالة: الاداليا، الطحالب البحرية، كبريتات الزنك، الرش الورقي،الدرنات.
} 
تعد الداليا. D.hybrida من نباتات الزينة المهمة في العالم وهي تتبع الى العائلة المركبة Asteraceae التي تضم . ... ب نوع من النباتات.

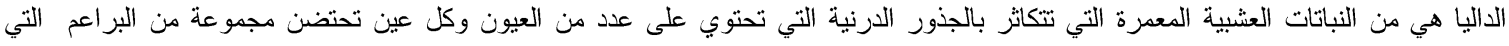

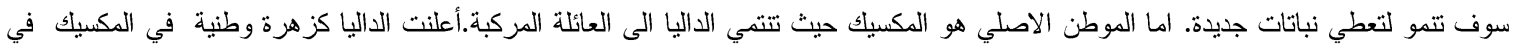

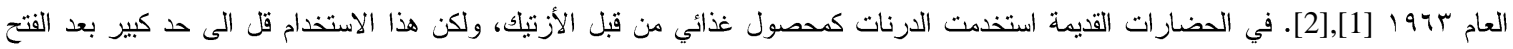

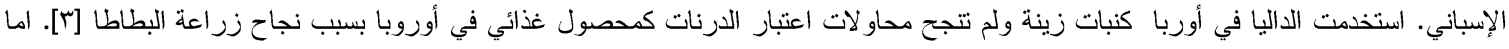
القيمة الغذائية للارنات في الوقت الحالي لاني تزال الداليا تعد واحدة من المكونات الغذائية الاساسية في المطبخ لكل من شعوب المكسيك وكولومبيا، اذ تزرع عدة أصناف لاستخدام درناتها الكبيرة مثل البطاطا الحلوة, او استخدام مستخلص الدرنات المحمصة في نكهات المشروبات في جميع أنحاء

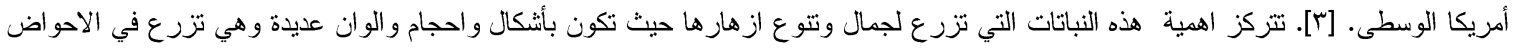

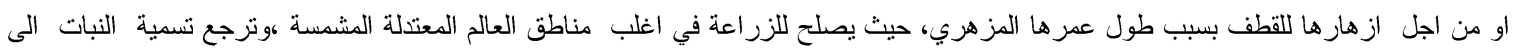

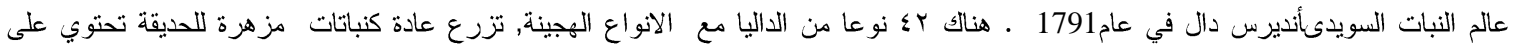

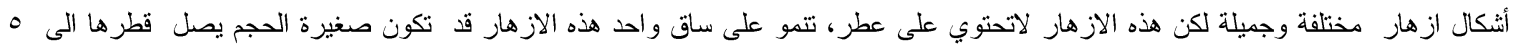

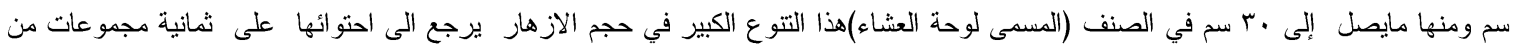
الكروموسومات المتمائلة، في حين أن معظم النباتات تحتوي على الثين فقط بالإضافة إلى ذلك، تحتوي الداليا أيضا على العديد من القطع الناقلة الجينية

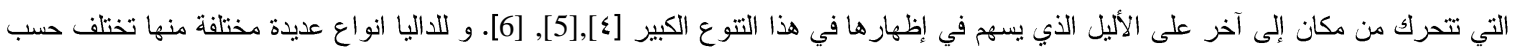

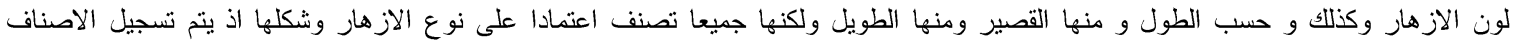

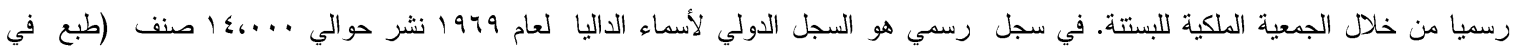

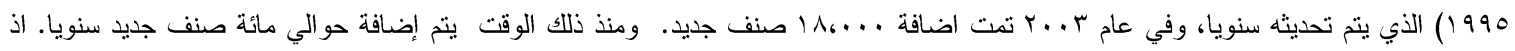
تم التصنيف اعتمادا على نوع الزهرة وقد قسم اصناف الداليا الى أربع عشرة مجموعة، جنبا إلى جنب مع الاختصار ات المستخدمة من قبل

.[9]،[8]،[7] (RHS)

اثتتت الدراسات بان الستخدام التغذية الورقية بالرش له تأثير في النمو الخضري والزهري لنباتات الزينة المختلفة حيث تلعب دور هاما في العمليات الحيوية والفسلجية داخل النبات كالتنفس والتمثيل الضوئي وانتاج الطاقة والتفاعلات الانزيمية بالإضافة الى دورها في كفاءة نقل نو اتج التمثيل

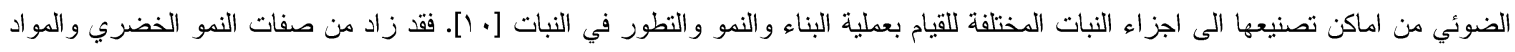

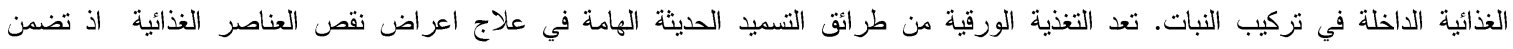

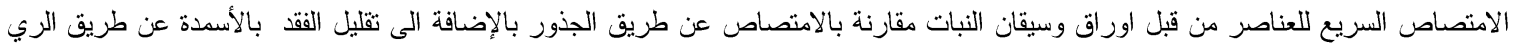

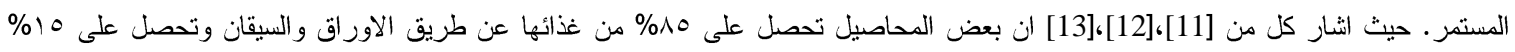

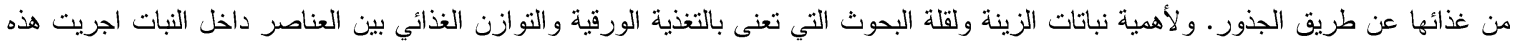

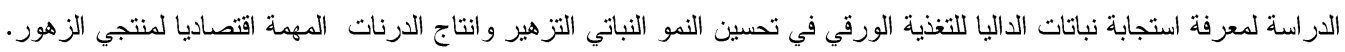

المواد وطر ائق العمل

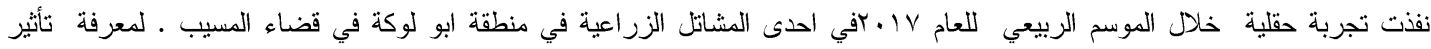

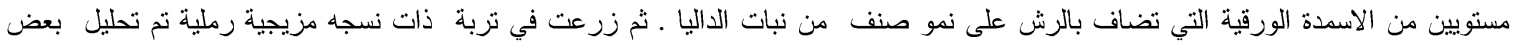

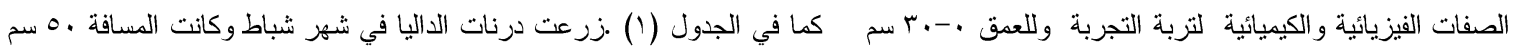

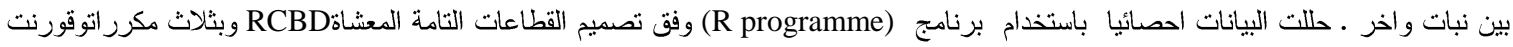

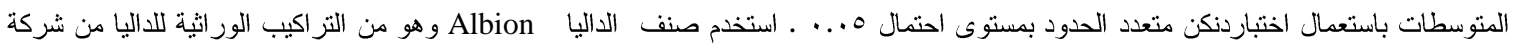
Florex

المعاملات

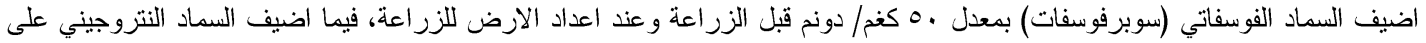

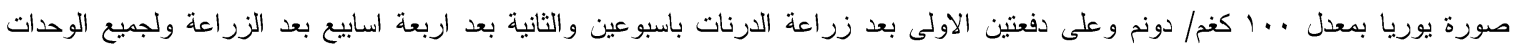

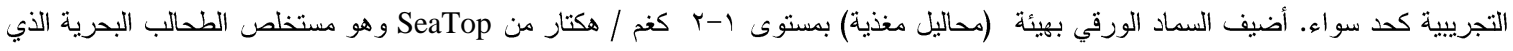

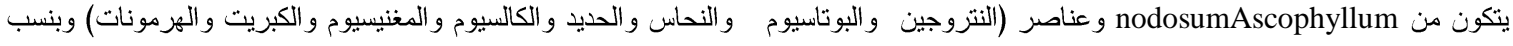

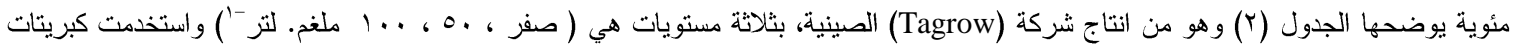




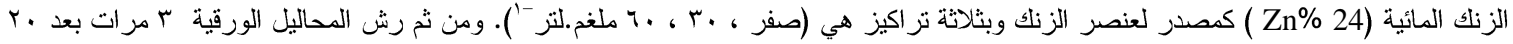

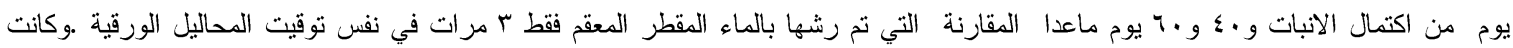

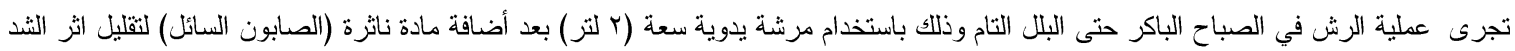

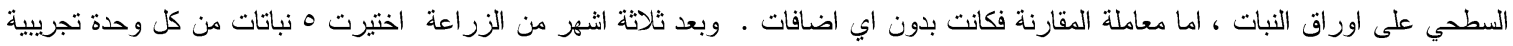
لقياس الصفات المدروسة الخاصة بمؤشرات النمو الخضري ثم حصدت الاجزاء الخضرية للنبات من منطقة تلامسها مع سطح التربة وتم قلع الدرنات

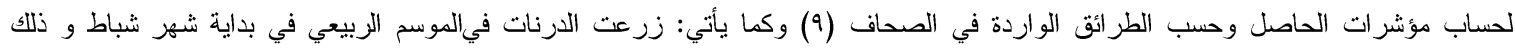

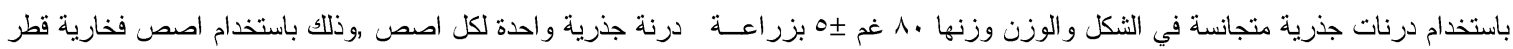
. با سم حاوية على 10 كغم من تربة ذات نسجه رملية مزيجية.

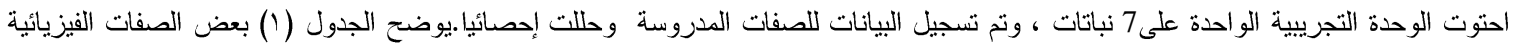

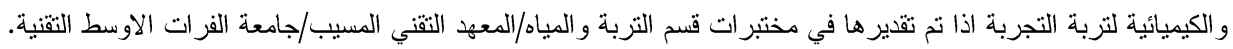

جدول (1) يوضح بعض صفات تربة الدراسة Characteristics of study soil

\begin{tabular}{|c|c|c|}
\hline القياس & الوحدة & الخاصية \\
\hline 7.2 & & درجة تفاعل التربة pH \\
\hline 2.8 & ديسي سيمنز م -1 & الايصالية الكهربائية (1:1) ECe \\
\hline 16 & سنتيمول. كفم -1 & السعة التبادلية الايونية الموجبة CEC \\
\hline 7.0 & \multirow{6}{*}{ غم .كغم 1- } & المادة العضوية \\
\hline 18.1 & & كاربونات الكالسيوم \\
\hline 110 & & الطين \\
\hline 240 & & الغزين \\
\hline 650 & & الرمل \\
\hline رملية مزيجة & & النسجة \\
\hline 1.3 & ميكا غم .م-" & الكثافة الظاهرية \\
\hline
\end{tabular}

جدول (Y): يوضح مكونات العماد الورقي للطحالب البحرية (W (W) (W)

\begin{tabular}{|c|c|}
\hline & $\%(\mathrm{w} / \mathrm{w})$ \\
\hline Appearance & 16 \\
\hline Alginic Acid & 50 \\
\hline Organic Matter & 1 \\
\hline $\mathbf{N}$ & $16-21$ \\
\hline K2O & $600-800 p p m$ \\
\hline Cytokinin\& gibberellin & $1-6$ \\
\hline Mannitol & 0.2 \\
\hline Fe & 0.15 \\
\hline Ca & 0.2 \\
\hline Mg & 1 \\
\hline S & 100 \\
\hline Solubility &
\end{tabular}

الصفات المدروسة

الصفات المدروسة/ مؤشرات النمو الخضري و الزهري و الحاصل :اختيرت خمسة نباتات عشو ائيا من كل وحدة تجريبية ووضعت علامات دالة عليها لغرض تسجيل البيانات لمؤشرات النمو الخضري عند بداية التزهير وصفات الحاصل عند النضج التام. 
صفات النمو الخضري و الحاصل ومكوناته: ا- طول النبات (سم): قبس هذا المؤشر في نهاية موسم النمو من منطقة اتصال الساق بالتربة الى القمة النامية للنبات بوساطة الثريط المتري. ץ- عدد الافرع لكل نبات-' حسبت عدد الافرع الرئيسة لكل نبات من نباتات الوحدة التجريبية.

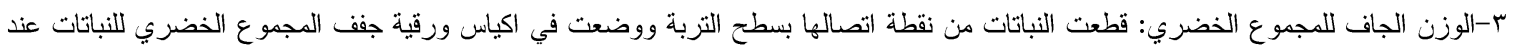

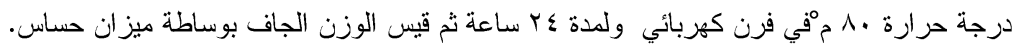

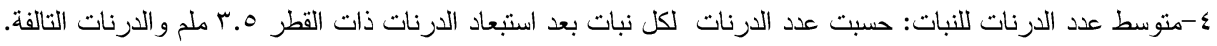
ه-متوسط وزن الدرنة: وزنت الدرنات لكل نبات بميزان كهربائي حساس.

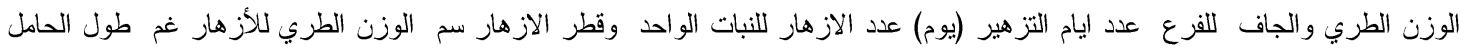

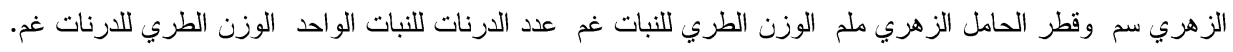

حلت نتائج التجربة احصائيا باستخدام برنامج ار (R programme) وتمت مقارنة بين المتوسطات الحسابية للمعاملات حسب اختبار دنكن

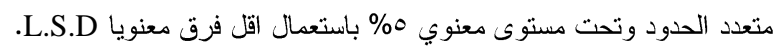

النتائج و المناقشة

صفات النمو الخضري

يوضح من الجدول ب وجود فروقات معنوية بين مستويات رش الطحالب البحرية في التأثير في صفات متوسط طول النبات وعدد الافرع في الني

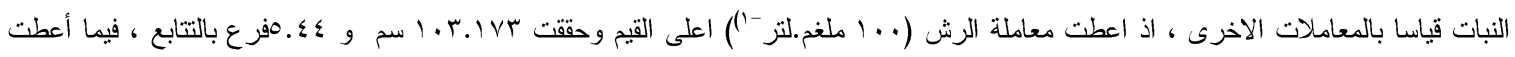

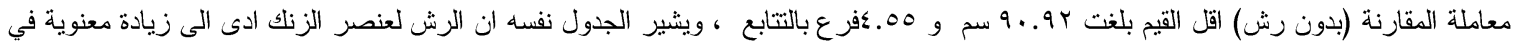

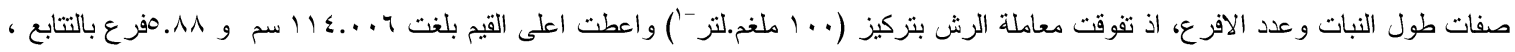

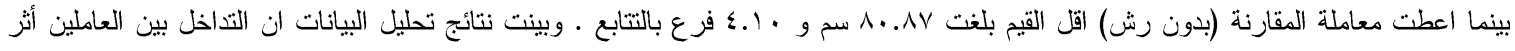

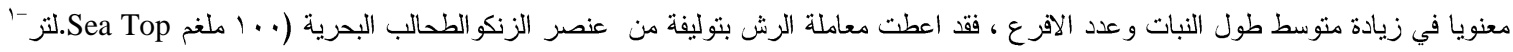

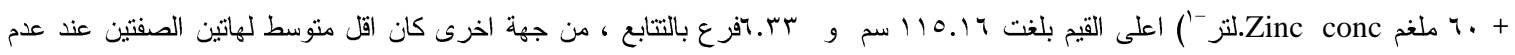

$$
\text { الرش بهما. }
$$

جدول رقم (r): يوضح تأثير الرش الورقي في صفات النبات الخضرية وطول النبات سم وعدد الافرع في نبات الداليا صنف Albion

\begin{tabular}{|c|c|c|c|c|c|c|c|c|}
\hline \multirow{2}{*}{$\begin{array}{c}\text { المتوسط } \\
\text { Average }\end{array}$} & \multicolumn{3}{|c|}{ 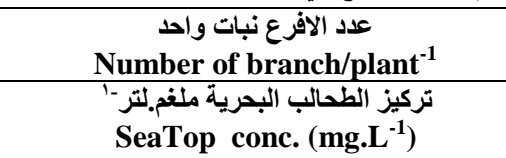 } & \multirow{2}{*}{$\begin{array}{c}\text { المتوسط } \\
\text { Average }\end{array}$} & & $\begin{array}{l}\text { ل النبات (سد) الب البحرية مt height } \\
\text { p conc. ( }\end{array}$ & & \multirow{2}{*}{ 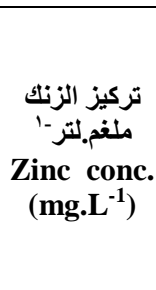 } \\
\hline & 100 & 50 & . & & 100 & 50 & . & \\
\hline 4.106 & $4.66 \mathrm{bc}$ & $4.33 \mathrm{bc}$ & 3.33 c & 80.87 & 83.53 c & $83.66 \mathrm{c}$ & $75.43 \mathrm{~d}$ & . \\
\hline 5.106 & $5.33 \mathrm{ab}$ & $5.33 \mathrm{ab}$ & $4.66 \mathrm{bc}$ & 96.77 & $110.83 \mathrm{a}$ & $95.26 \mathrm{~b}$ & 84.23 c & 30 \\
\hline 5.883 & 6.33 a & $5.66 \mathrm{ab}$ & 5.66ab & 114.00 & $115.16 \mathrm{a}$ & 113.76a & $113.10 \mathrm{a}$ & 60 \\
\hline & 5.44 & 5.106 & 4.55 & & 103.173 & 97.56 & 90.92 & $\begin{array}{c}\text { المتوسط } \\
\text { Average }\end{array}$ \\
\hline
\end{tabular}

p p القيم ذات الاحرف المتشابهة لكل عامل او تداخلاتها كل على انفر اد لا تختلف معنوياً حسب اختبار دنكن متعدد الحدود تحت مستوى احتمال 
جدول رقم (ع): يوضح تأثير الرش الورقي في صفات النبات الخضرية عدد الازهار في النبات الواحد وقطر الزهرة سم فينبات الاداليا صنف Albion

\begin{tabular}{|c|c|c|c|c|c|c|c|c|}
\hline \multirow{3}{*}{$\begin{array}{c}\text { المتوسط } \\
\text { Average }\end{array}$} & \multicolumn{3}{|c|}{ 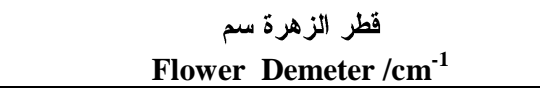 } & \multirow{3}{*}{ المتوسط } & \multicolumn{3}{|c|}{$\begin{array}{c}\text { عدد الازهار في النبات الواحد } 1 \text { عار /plant } \\
\text { Number of flower /plant }\end{array}$} & \multirow{3}{*}{ 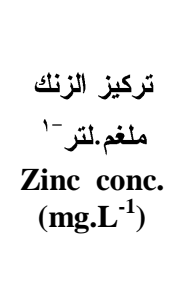 } \\
\hline & \multicolumn{3}{|c|}{ 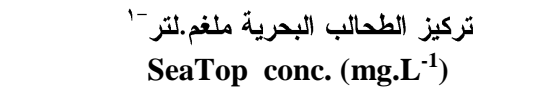 } & & \multicolumn{3}{|c|}{$\begin{array}{l}\text { - تركيز (الطحالب البحرية ملفم.لتر } \\
\text { SeaTop conc. (mg.L }{ }^{-1} \text { ) }\end{array}$} & \\
\hline & 100 & 50 & $\mathbf{0}$ & & 100 & 50 & $\mathbf{0}$ & \\
\hline 11.74 & 13.16ab & 11.80bc & $9.93 \mathrm{c}$ & 8.776 & 10.00ab & $9.33 \mathrm{bc}$ & $7.00 \mathrm{c}$ & 0 \\
\hline Ir.AV & 13.83ab & 13.26ab & $11.53 \mathrm{bc}$ & 9.996 & 10.33ab & 10.00ab & $9.66 \mathrm{ab}$ & 30 \\
\hline $1 \leqslant . V V$ & $15.53 \mathrm{a}$ & $15.23 \mathrm{a}$ & $13.56 \mathrm{ab}$ & 11.443 & $12.00 \mathrm{a}$ & 11.33ab & $11.00 \mathrm{ab}$ & 60 \\
\hline & 14.17 & 13.43 & 11.67 & & 10.77 & 10.22 & 9.22 & $\begin{array}{l}\text { المتوسط } \\
\text { Average }\end{array}$ \\
\hline
\end{tabular}

p>0.05 القىم ذات الأحرف المتشاب هت لكل عامل لا تختلف معنوىاً حسب اختبار دنكن متعدد الحدود تحت مستوى احتمال

يبين الجدول ؛ وجود فروقات معنوية بين مستويات رش الطحالب البحرية في التأثير في صفات متوسط عدد الازهار في النبات الواحد وقطر

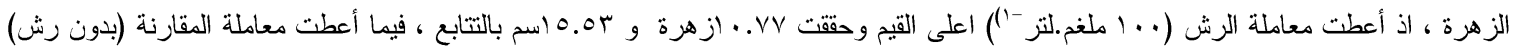

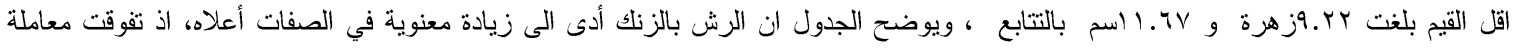

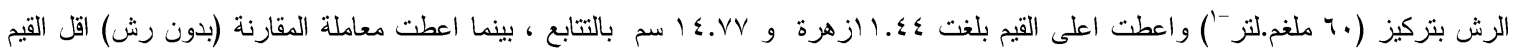

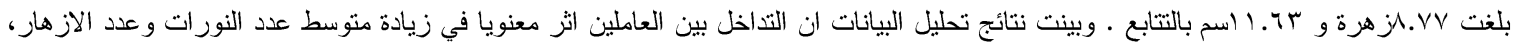

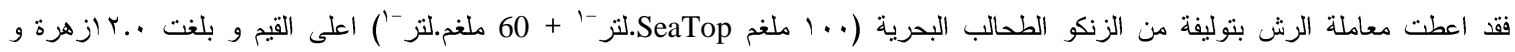

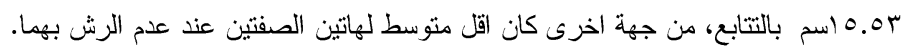

جدول رقم (•): يوضح تأثير الرش الورقي في صفات النبات الخضرية الوزن الطري والجاف في نبات الداليا صنف Albion .

\begin{tabular}{|c|c|c|c|c|c|c|c|c|}
\hline \multirow{3}{*}{$\begin{array}{c}\text { المتوسط } \\
\text { Average }\end{array}$} & \multirow{2}{*}{\multicolumn{3}{|c|}{ 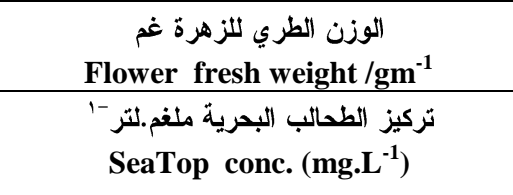 }} & \multirow{3}{*}{ المتوسط } & \multirow{2}{*}{\multicolumn{3}{|c|}{ 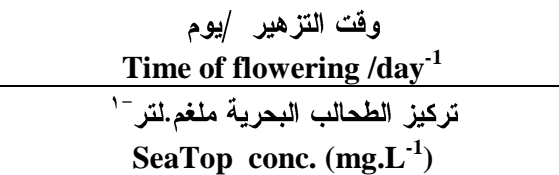 }} & \multirow{3}{*}{$\begin{array}{c}\text { تركيز الزنت ملفم.لتر-1 } \\
\text { Zinc conc. } \\
\text { (mg.L }{ }^{-1} \text { ) }\end{array}$} \\
\hline & & & & & & & & \\
\hline & 10 . & 50 & 0 & & 100 & 50 & 0 & \\
\hline 24.95 & 25.66 a & 24.53ab & 24.66ab & 116.42 & $116.40 \mathrm{a}$ & $116.33 \mathrm{a}$ & $116.53 \mathrm{a}$ & 60 \\
\hline & 24.506 & 23.81 & 22.54 & & 113.68 & 113.37 & 1113.7 & $\begin{array}{c}\text { المتوسط } \\
\text { Average }\end{array}$ \\
\hline
\end{tabular}

p القيم ذات الأحرف المتتاب هة لكل عامل لا تختلف معنوىاً حسب اختبار دنكن متعدد الحدود تحت مستوى احتمال 0.05

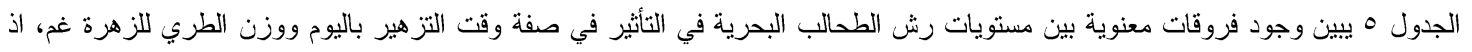

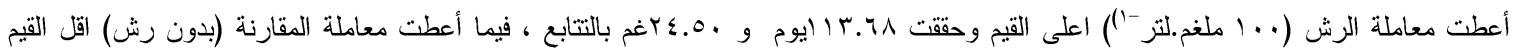

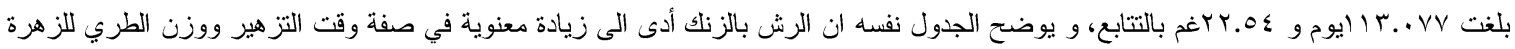

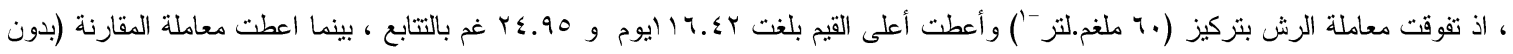

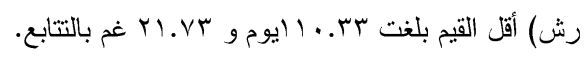


وبينت نتائج تحليل البيانات ان التداخل بين العاملين أثز معنويا في زيادة متوسط وقت التزهير ووزن الطري/غم، فقد أعطت معاملة الرش بتوليفة

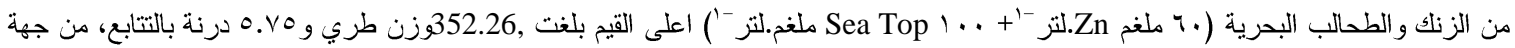
اخرى كان اقل متوسط لهاتين الصفتين عند عدم الرش بهما.

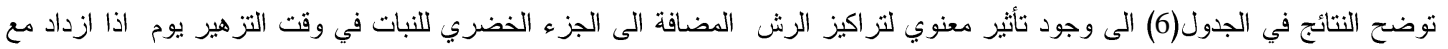

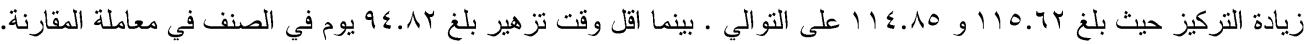

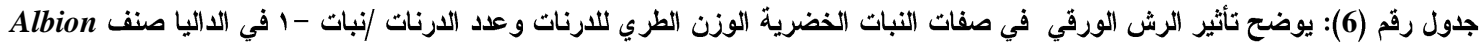

\begin{tabular}{|c|c|c|c|c|c|c|c|c|}
\hline \multirow{2}{*}{ المتوسط } & \multicolumn{3}{|c|}{ 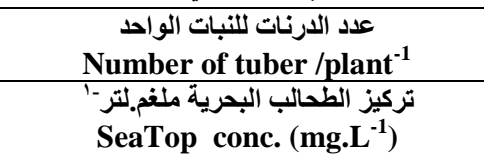 } & \multirow{2}{*}{$\begin{array}{c}\text { المتوسط } \\
\text { Average }\end{array}$} & \multicolumn{3}{|c|}{ 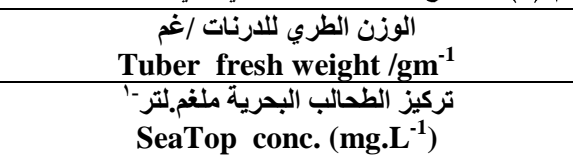 } & \multirow{2}{*}{ 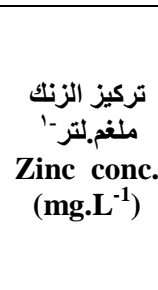 } \\
\hline & 100 & 50 & 0 & & 100 & 50 & 0 & \\
\hline 6.606 & $6.66 \mathrm{a}$ & $6.50 \mathrm{a}$ & $6.66 \mathrm{a}$ & 365.356 & 371.36 a & 361.11ab & 363.60ab & 60 \\
\hline & 5.75 & 5.6 & 4.886 & & 352.263 & 347.523 & 326.876 & $\begin{array}{c}\text { المتوسط } \\
\text { Average }\end{array}$ \\
\hline
\end{tabular}

p<0.05. القيم ذات الأحرف المنتاب هزة لكل عامل لا تختلف معنوىاً حسب اختبار دنكن منعدد الحدود تحت مستوى احتمال

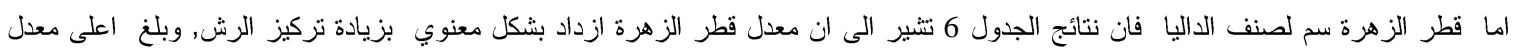

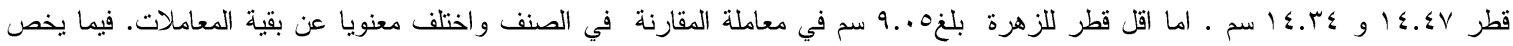
استجابة صنف الداليا لزيادة قطر الزهرة.

جدول رقم (7): يوضح ثأثير الرش الورفي في صفات النبات الخضرية الوزن الطري للحامل الزهري وطول الحامل الزهزي /سمفي نبات الداليا صنف Albion

\begin{tabular}{|c|c|c|c|c|c|c|c|c|}
\hline \multirow{2}{*}{$\begin{array}{c}\text { المتوسط } \\
\text { Average }\end{array}$} & \multicolumn{3}{|c|}{ 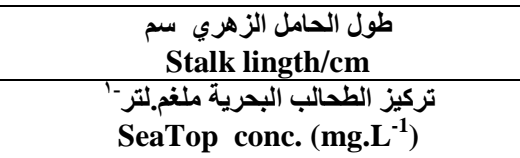 } & \multirow{2}{*}{ المتوسط } & \multicolumn{3}{|c|}{$\begin{array}{l}\text { الوزن الطري للحامل الزهري /غو /gm weight } \\
\text { Stalk fresh we }\end{array}$} & \multirow{2}{*}{ 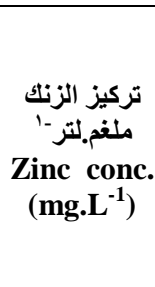 } \\
\hline & 100 & 50 & 0 & & 100 & 50 & 0 & \\
\hline 40.88 & 43.10a & 41.90ab & 37.66 b & 38.92 & 40.96 bcd & $39.41 \mathrm{~cd}$ & $36.40 \mathrm{~d}$ & $\mathbf{0}$ \\
\hline 45.68 & 46.06a & 45.43a & 45.56 a & 45.77 & 46.56 a & $45.66 \mathrm{ab}$ & 45.10ab & 60 \\
\hline & 44.30 & 43.5 & 42.15 & & 43.37 & 42.15 & 40.86 & $\begin{array}{c}\text { المتوسط } \\
\text { Average }\end{array}$ \\
\hline
\end{tabular}

p<0.05. القيم ذات الأحرف المتتـاب هة لكل عامل لا تختلف معنوىاً حسب اختبار دنكن متعدد الحدودتحت مستوى احتمال

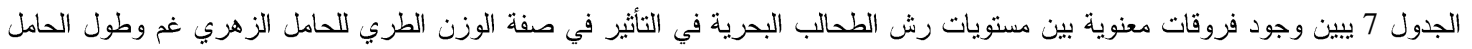

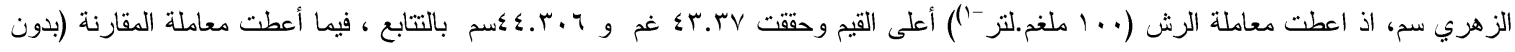

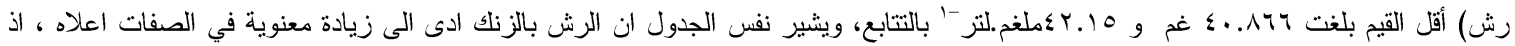

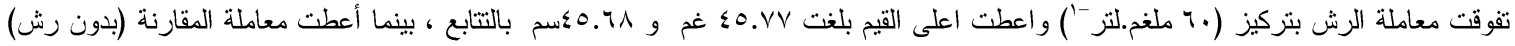

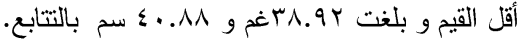


جدول رقم (^): يبين تأثير الرش الورقي في صفات النبات الخضرية قطر الساق ملم /الوزن الجاف للجزء الخضري في نبات الداليا صنف Albion

\begin{tabular}{|c|c|c|c|c|c|c|c|c|}
\hline \multirow{3}{*}{$\begin{array}{c}\text { المتوسط } \\
\text { Average }\end{array}$} & \multirow{2}{*}{\multicolumn{3}{|c|}{ 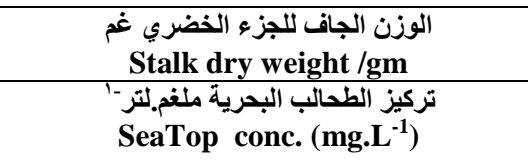 }} & \multirow{3}{*}{$\begin{array}{c}\text { المتوسط } \\
\text { Average }\end{array}$} & \multirow{2}{*}{\multicolumn{3}{|c|}{ 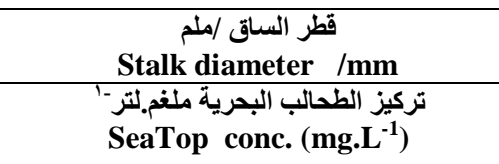 }} & \multirow{3}{*}{ 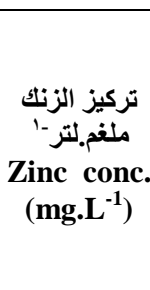 } \\
\hline & & & & & & & & \\
\hline & 100 & 50 & O & & 100 & 50 & 0 & \\
\hline 60.04 & $60.16 a$ & 60.06a & 59.90 a & 6.45 & 6.66a & $6.43 \mathrm{a}$ & $6.26 \mathrm{a}$ & 60 \\
\hline & 55.73 & 52.55 & 51.24 & & 6.36 & 6.24 & 5.94 & $\begin{array}{c}\text { المتوسط } \\
\text { Average }\end{array}$ \\
\hline
\end{tabular}

0.05. القيم ذات الأحرف المتشاب هة لكل عامل لا تختلف معنوىاً حسب اختبار دنكن متعدد الحدود تحت مستوى احتمال

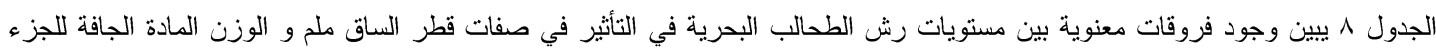

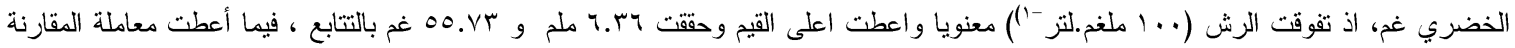
(بدون رش) اقل القيم بلغت ؟ 9.0

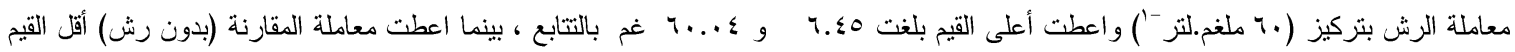

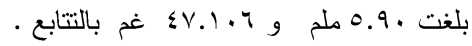

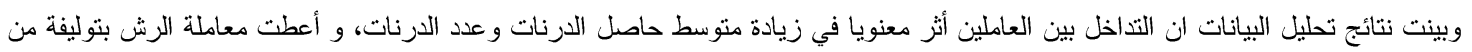

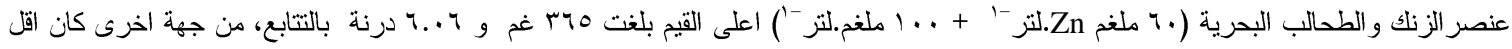

متوسط لهاتين الصفتين عند عدم الرش بهما.

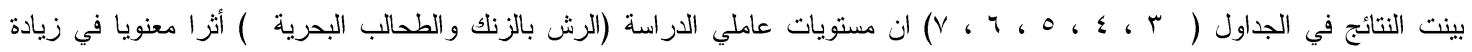

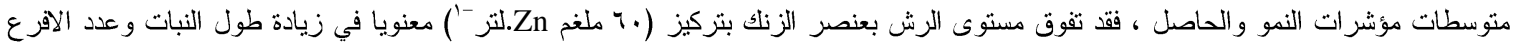

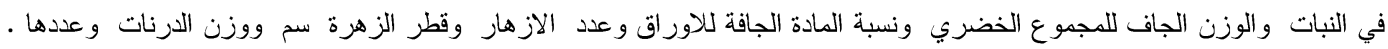

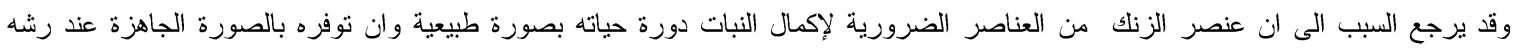

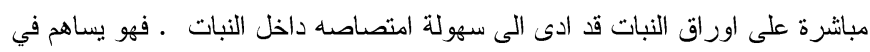

الكثير من العمليات الحيوية المختلفة التي تجري داخل النبات والتي تؤدي الى نمو جيد للنبات والى بناء مجموع جذري كثيف يزيد من كفاءة

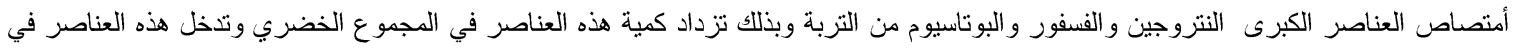

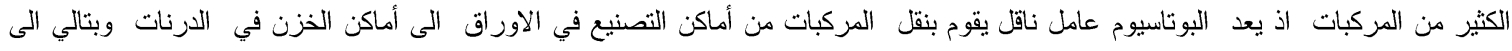

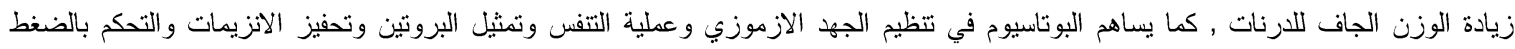

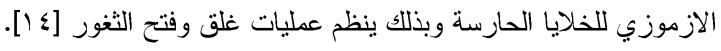

فضلا أن للزنك دورا في زيادة البروتين من خلال دوره في تكوين الحامض الاميني التربتوفان، كما ان له دورا في تكوين الـ RNA الضروري

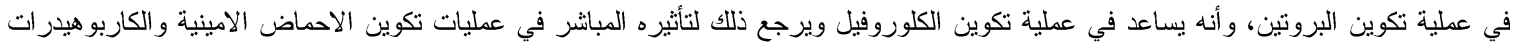

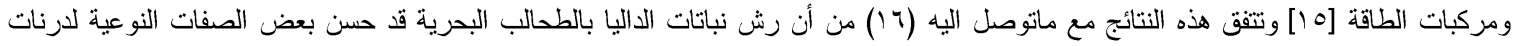

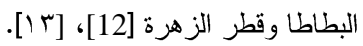

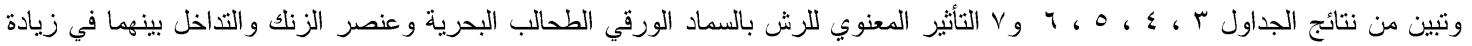
متوسطات الصفات المدروسة ( ارتفاع الساق الرئيسي وعدد الاوراق للنبات وعدد الافرع للنبات و الوزن الجاف للمجموع الخضري وعدد الدرنات

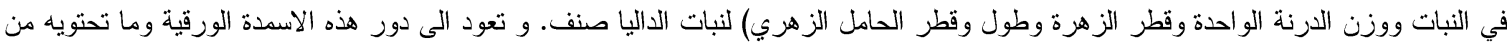
عناصر مغذية ومنها محلول الطحالب البحرية الذي يحتوي على العديد من العناصر المغذية والتي لها دور مباثر في عمليات التمنيل الضونئي وانقسام

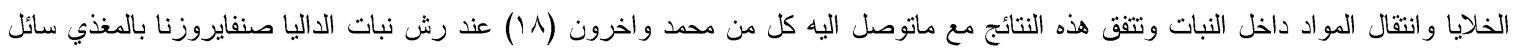
اسكوربيك اسد [17]،[18]،[19].عند رش سماد الحيوي على الداليا [ب 1]]. 
نسنتتج من هذه التجربة أهمية التخذية الورقية بالطحالب البحرية مع الزنك على نباتات الزينة، ان رش السماد الورقي الطحالب البحرية

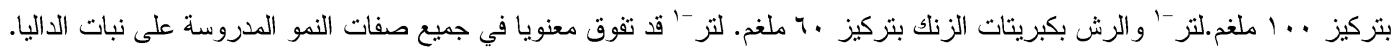

\section{CONFLICT OF INTERESTS}

There are no conflicts of interest.

[1] H. A. Howard, The Dahlia: An Early History, 1929.

[2] B. K. and I. K. Hughes, Growing Dahlias, Queensland Department of primary industries .Brisbane. Australia, 1985.

[3] H. V. Hansen, Native (wild) Dahlias - taxonomy, historical review, and the derivation of cultivars, in Trial of Dahlia, Final Report, Royal Horticultural Society.9om, 2008.

[4] R. Horticultural Society, Retrieved 19 June , 2015.

[5] W. Gerald, The Alpha-Omega of Dahlias, American Dahlia Society. Retrieved 2 July, 2015.

[6] D. E. Giannasi, The flavonoid systematics of the genus Dahlia (comstoopidpositae), Memoirs of the New York Botanical Garden, vol.26, no.2. New York Botanical Garden, 1975.

[7] Bates, Dahlia types and international classification of dahlias, 2015.

[8] P. D. Sørensen, New taxa in the genus Dahlia (Asteraceae, Heliantheae-Coreopsidinae). Rhodora, vol.82, pp.353360, 1980.

[9] R. Horticultural Society, The International Dahlia Register. Twenty Second Supplement, 2015.

[10] تعبان ,صادق كاظم، " ناثير اضافة السماد الورقي والارضي البوتاسيوم في نمو حاصل الحنطة . Triticumaestivum L".، رسالة ماجستير

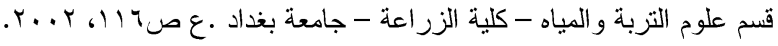

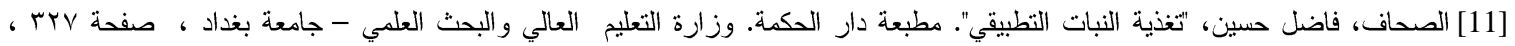

[12] M. A. Elsadek, "Improvement Yield and Quality of Dahlia Flowers by Exogenous Application of Gibberellic Acid and Salicylic Acid under Sandy Soil Conditions", J. Plant Production, Mansoura Univ., vol. 9, no.3, pp.289 -297, 2018.

[13] E.-Alsayed, G. Sohier, M. Sahar Ismail and D. Eissa , "Impact of Seaweed Extract and Phosphorus Application on Productivity of Dahlia Plants .Assiut", J. Agric. Sci., vol.49, no.1, pp.159-188, 2018.

[14] محمود، جواد طه، " تأثير رش نباتات البطاطا المسمدة عضويا بالحديد والزنك عند مراحل نمو مختلفة في بعض صفات الحاصل" ، مجلة

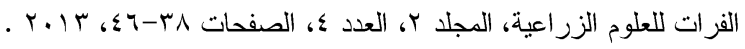

[15] ابو ضاحي ، يوسف محمد و مؤيد احمد اليونس ،. "دليل تغذية النبات" · جامعة بغداد ، وزارة التعليم العالي والبحث العلمي، العراق، صفحة

$1911, \leqslant 11$

[16] عبد العباس, فخرية عبد الله , الجابري مهدي نعمة , حسن فاطمة علي، "تاثير الرش بحامض الجبرلين وسائل جوز الهند في النمو الخضري

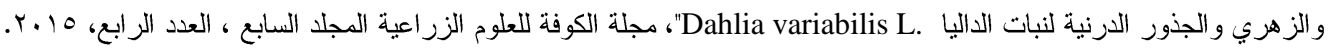

[17] N. d. manolyand abd-elmegide a. nasr.. "response of dahlia pinnata plants to biofertilizer types" .egypt.j. exp. biol. , vol.4, pp.87-91, 2008.

[18] سارة علي محمد و سوسن عبد الله عبد اللطيف واياد عاصي عبيد. "تاثير الرش الورقي بكبريتات البوتاسيوم وحامض الاسكوربيك في نمو

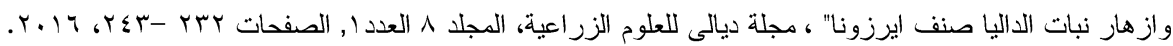

[19] A. Younis, S. Anjum, A. Riaz, M. Hameed, U. Tariq \& M. Ahsan, "Production of quality dahlia (Dahlia variabilis cv. Redskin) flowers by efficient nutrients management", American-Eurasian J Agric\& Environ Sci., vol.14, no.2, pp. 137-142, 2014. 